\title{
Characterization of the near-interface region of chemical vapor deposited diamond films on silicon by backscatter Kikuchi diffraction
}

\author{
Brent L. Adams \\ b_I_adams@byu.edu \\ K. Kunze \\ S. Geier \\ R. Hessmer \\ M. Schreck \\ Follow this and additional works at: https://scholarsarchive.byu.edu/facpub \\ o next page for additional authors \\ Part of the Mechanical Engineering Commons
}

\section{Original Publication Citation}

Geier, S., M. Schreck, R. Hessmer, B. Rauschenbach, B. Stritzker, K. Kunze, and B. L. Adams. "Characterization of the near-interface region of chemical vapor deposited diamond films on silicon by backscatter Kikuchi diffraction." Applied Physics Letters 6

\section{BYU ScholarsArchive Citation}

Adams, Brent L.; Kunze, K.; Geier, S.; Hessmer, R.; Schreck, M.; Rauschembach, B.; and Stritzker, B., "Characterization of the near-interface region of chemical vapor deposited diamond films on silicon by backscatter Kikuchi diffraction" (1994). Faculty Publications. 694.

https://scholarsarchive.byu.edu/facpub/694

This Peer-Reviewed Article is brought to you for free and open access by BYU ScholarsArchive. It has been accepted for inclusion in Faculty Publications by an authorized administrator of BYU ScholarsArchive. For more information, please contact ellen_amatangelo@byu.edu. 


\section{Authors}

Brent L. Adams, K. Kunze, S. Geier, R. Hessmer, M. Schreck, B. Rauschembach, and B. Stritzker 


\title{
Characterization of the near-interface region of chemical vapor deposited diamond films on silicon by backscatter Kikuchi diffraction
}

\author{
S. Geier, M. Schreck, R. Hessmer, B. Rauschenbach, and B. Stritzker \\ Institut für Physik, Universität Augsburg, D-86135 Augsburg, Germany \\ K. Kunze ${ }^{\text {a) }}$ and B. L. Adams \\ Manufacturing Engineering Department, Brigham Young University, Provo, Utah 84602
}

(Received 4 January 1994; accepted for publication 12 July 1994)

\begin{abstract}
The lattice orientations near the interface of chemical vapor deposited diamond films on $\mathrm{Si}(001)$ have been studied by orientation imaging microscopy. This technique is based on the automated analysis of electron backscatter Kikuchi diffraction patterns. The electron beam has been scanned in discrete steps over the reverse side of the diamond film after having removed the substrate. The obtained data have allowed us to determine the texture and to visualize quantitatively the orientational arrangement of and among individual diamond crystallites in the near-interface region. A comparison with the orientation of the substrate has proved the existence of epitaxially nucleated grains. A high amount of twinned diamond has been deduced from the pole figures and verified by analysis of orientation correlations between neighboring crystallites. Moreover, the grain boundary maps have allowed us to monitor and quantify directly the occurring twin boundaries. (C) 1994 American Institute of Physics.
\end{abstract}

Intensive research activities are currently aimed at growing high quality diamond films by chemical vapor deposition (CVD). Especially for heteroepitaxial deposition on economically interesting substrates like silicon it is necessary to gain a better understanding of the diamond's nucleation and growth characteristic. Studies by cross-sectional transmission electron microscopy (XTEM) ${ }^{1,2}$ and high resolution $\mathrm{TEM}^{3,4}$ have been performed to characterize the interface and internal defects of diamond films which have been deposited on various substrates. These techniques for the study of orientation relationships are limited to measurements in small regions. Sample preparation as well as the evaluation of statistically evident texture data are quite laborious.

On the other hand research groups ${ }^{5-8}$ have employed $\mathrm{x}$-ray diffraction (XRD) pole figure measurements to characterize the film quality with respect to preferential orientations of the crystallites. Because of its integrating character this technique yields low depth and lateral resolution. Therefore studies on the texture formation in polycrystalline diamond films are performed by measuring pole figures as a function of the film thickness. ${ }^{6}$ The texture in a certain depth of the film can then be deduced indirectly by comparing the texture measurements for two subsequent film thicknesses. It would be highly desirable to measure the texture directly with high depth resolution. Further, this would allow the measurement of the texture at the interface. When an evolutionary growth process follows an epitaxial nucleation step, ${ }^{9}$ neither all of the grains are oriented nor is the orientation with respect to the substrate perfect. Especially in the latter case some knowledge about the texture at the interface would be helpful for further optimizing the film quality.

The analysis of electron backscattering patterns [also known as backscatter Kikuchi diffraction (BKD) patterns] in

\footnotetext{
a) Present address: Institut für Metallkunde and Metallphysik, TU Clausthal, D-38678 Clausthal-Zellerfeld, Germany.
}

the scanning electron microscope (SEM) has been introduced by Venables and Harland. ${ }^{10}$ Dingley and Baba-Kishi ${ }^{11}$ have used the on-line analysis of BKD for local crystallography. Recently a completely automated system which couples automatic analysis of BKD patterns with precise positioning of the sample in the SEM has been developed. ${ }^{12}$ This technique called orientation imaging microscopy (OIM) allows the determination of lattice orientation at many laterally different locations in the near-surface region of the specimen. The lateral resolution has been estimated below $1 \mu \mathrm{m}$, and is restricted by the probe diameter (size of the interaction volume) of $0.5 \mu \mathrm{m}$ as well as the minimum reproducible step size of $0.25 \mu \mathrm{m}$. The information depth for diamond is about $40 \mathrm{~nm}^{13}$

We have employed the OIM technique for the investigation of diamond films which have been grown in a commercial microwave plasma CVD reactor. A bias pretreatment has been used to nucelate diamond on the $\mathrm{Si}(001)$ substrate. This step has been followed by a growth process at $770{ }^{\circ} \mathrm{C}, 900$ $\mathrm{W}$, and $0.5 \% \mathrm{CH}_{4}$ in $\mathrm{H}_{2} \cdot{ }^{8}$ We have chosen a relatively low diamond nucleation density of about 1 nucleus per $10 \times 10$ $\mu \mathrm{m}^{2}$. This value had been determined by interrupting the process after the nucleation step and measuring the density of the submicron diamond crystallites on the silicon surface by SEM. The substrate has been partially removed by chemically etching a hole of $5 \mathrm{~mm}$ diameter into the silicon wafer with $\mathrm{HF} / \mathrm{HNO}_{3}$. Before that the diamond film has been covered from the top with silver epoxy and attached to a silicon wafer in order to stabilize the film at the etched region.

The specimen is mounted in the SEM with a tilt angle of $70^{\circ}$ against the electron beam $(15 \mathrm{keV})$. The interaction of the incident beam with the sample produces backscattered electrons which are detected on a fluorescent screen mounted in the vacuum chamber. A high gain video camera transmits this image to the computer through a video digitizing board. The diffraction image is analyzed by the computer and the 


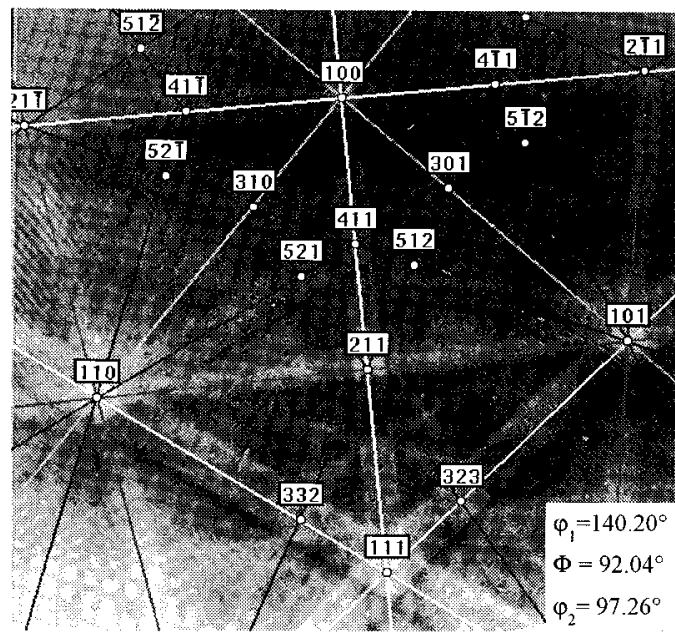

FIG. 1. Indexed BKD pattern taken from diamond at the diamond/silicon interface. The resulting Eulerian angles $\left(\varphi_{1}, \Phi, \varphi_{2}\right)$ determine the orientation of the crystal lattice of diamond at the position of the incident electron probe, relative to the microscope system.

orientation of the crystal lattice at the beam location is obtained. The computer also controls the movement of the specimen stage in the SEM. The scanning of the sample allows the automatic measurement of the orientation at each individual point. For analysis of electron backscattering patterns and further experimental details on OIM see Refs. 12 and 14 .

A BKD pattern of Si has been taken to determine the orientation of the substrate relative to the microscope system as a reference. Then, the electron beam was directed to the diamond film, where the substrate had been removed. Figure 1 displays an indexed BKD pattern of diamond which contains information about the orientation of the crystal lattice at the local region the electron beam has been directed to. The information depth of about $40 \mathrm{~nm}$ enables us to characterize the orientations of the diamond lattice in the near-interface region. Since every single measurement yields the complete set of Eulerian angles $\left(\varphi_{1}, \Phi, \varphi_{2}\right)$ any mode of representing the orientational arrangement, e.g., the orientation distribution function, the pole figure of any given reflex or a map imaging the measured orientations over a certain area, can be deduced directly from these data. The Eulerian angles are determined with an error of less than $1^{\circ}$.

Figure 2(a) displays a $\{111\}$ pole figure which is based on 6400 single orientation measurements. The measurements covered an area of $800 \times 800 \mu \mathrm{m}^{2}$ using a step size of $10 \mu \mathrm{m}$ on a regular square grid. By comparing with the corresponding pole diagram of the substrate [Fig. 2(b)] one recognizes that the $\{111\}$ pole density maxima of diamond have the same orientation as the $\{111\}$ poles of silicon. Even though the maxima are strongly broadened this proves the epitaxial relationship of diamond crystallites relative to the substrate. The weaker maxima in the pole figure can be explained by twinning on $\{111\}$ faces of epitaxially aligned diamond grains. For details see Ref. 8.

In order to visualize the twin boundaries we have performed an automatic orientation measurement over an area of $60 \times 60 \mu \mathrm{m}^{2}$ using a step size of $0.4 \mu \mathrm{m}$. Figure 3 illus-

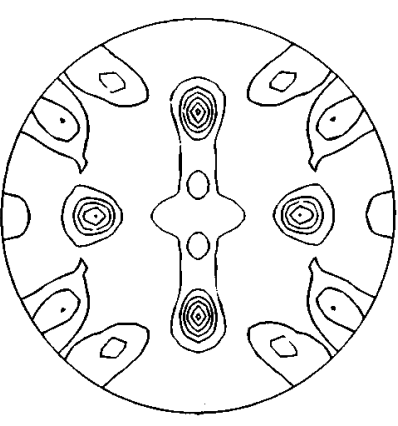

(a)

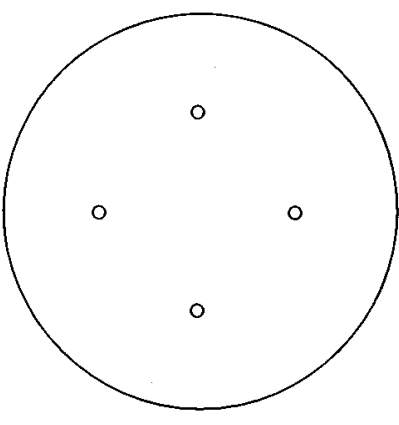

(b)
FIG. 2. (a) $\{111\}$ pole figure of the near-interface region of diamond. The single orientation measurements have covered an area of $800 \times 800 \mu \mathrm{m}^{2}$ using a step size of $10 \mu \mathrm{m}$. Contour levels: 1.0, 1.5, 2.0, 2.5, 3.0, 3.5. (b) $\{111\}$ pole diagram of the silicon substrate.

trates the resulting map of boundaries. The reconstruction of this map from 22500 single orientation measurements has been performed by simply comparing the measured orientations between neighboring points. If the orientation distance ${ }^{15}$ between two neighboring points exceeds $5^{\circ}$ a thin boundary line segment is drawn. A thick boundary line segment is drawn if the orientation difference ${ }^{15}$ corresponds to the relation which is expected for twin boundaries (within a tolerance range of $8.66^{\circ}$ according to Brandon's criterium in the CSL model). ${ }^{16}$ For diamond, twinning results in a rota-

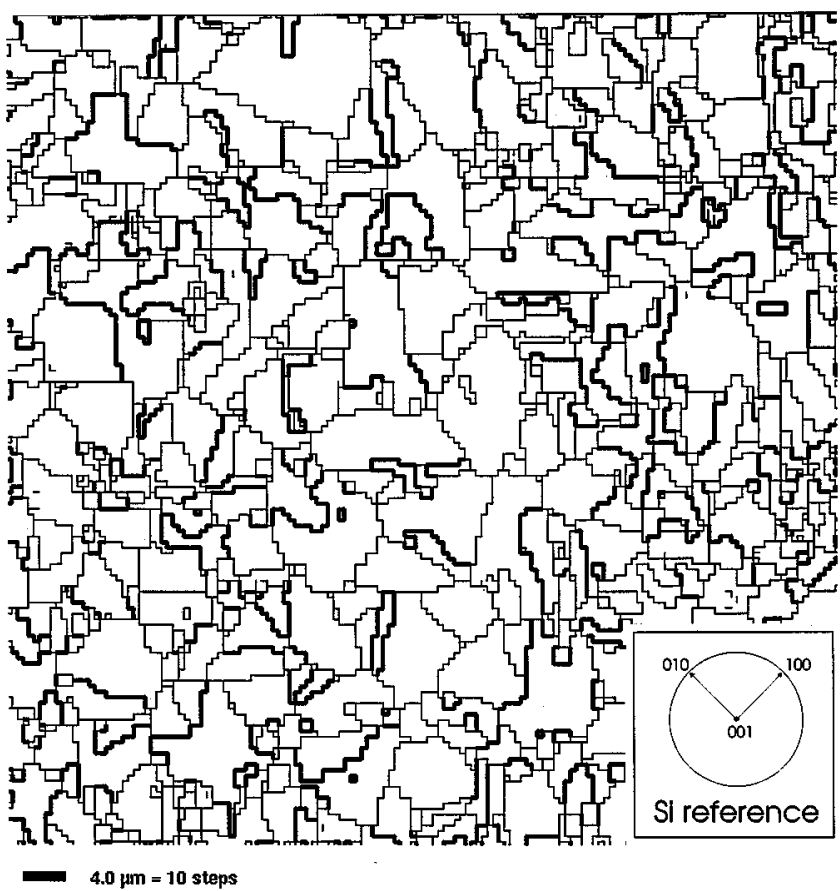

FIG. 3. Grain boundary map reconstructed from automatic orientation measurements on diamond at the diamond/silicon interface over an area of $60 \times 60 \mu^{2}$ using a step size of $0.4 \mu \mathrm{m}$. Thin boundary line segments correspond to orientation distances greater than $5^{\circ}$ between neighboring points. Thick boundary line segments are drawn if the orientation difference corresponds to twin boundaries. The inset shows the crystallographic orientation of the Si substrate relative to this map. 


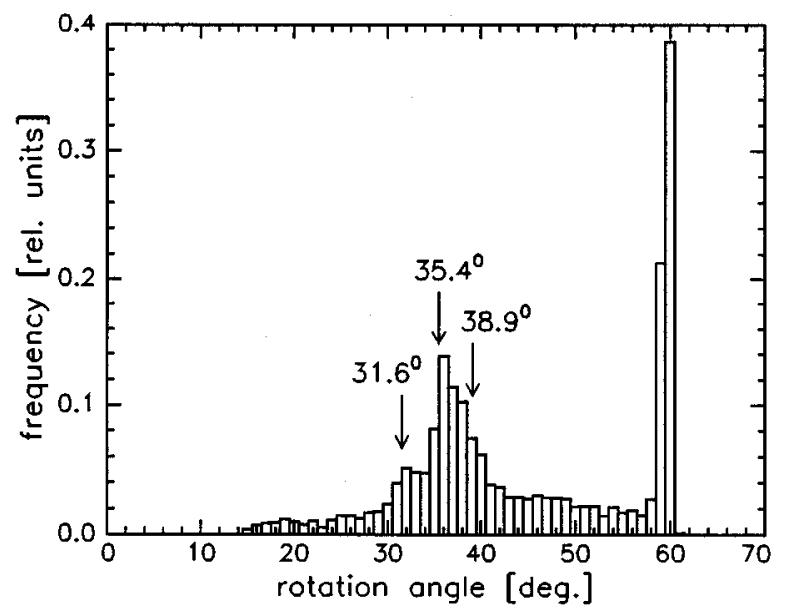

FIG. 4. (a) Frequency distribution of the rotation angle as deduced from the data of Fig. 3.

tion of $60^{\circ}$ around the $\langle 111\rangle$ axis ( $\Sigma 3$-type boundary). In contrast to the low nucleation density of about $0.01 \mu \mathrm{m}^{-2}$ the mean distance of the grain boundaries in Fig. 3 is clearly below $10 \mu \mathrm{m}$. Additional nucleation of diamond on silicon during the growth step can be neglected. As a consequence, besides boundaries which develop when different crystallites meet each other during the growth towards a closed film, a high amount of boundaries may only result from growth defects, e.g., the formation of twins.

From the data of the single orientation measurements of Fig. 3, those orientation differences between all nearest neighbors have been extracted which have a rotation angle greater than $15^{\circ}$ and thus correspond to large angle grain boundaries. Small angle grain boundaries are not intended to be studied in this letter. Figure 4 displays the frequency distribution of the rotation angle as deduced from our data. For a sample with a random orientation distribution and completely random neighbor correlations a rather smooth frequency distribution would be expected. ${ }^{17}$ In Fig. 4 the strongest peak at a rotation angle of $60^{\circ}$ corresponds to primary twinning with a $\langle 111\rangle$ rotation axis. The broad structure at about $36^{\circ}$ can be explained by higher order twinning. An angle of $38.9^{\circ}$ may result from the orientation difference of two neighboring measurement points which are separated by either one $\Sigma=9$ boundary or two different $\Sigma=3$ boundaries. Changing the selected $\langle 111\rangle$ axis after each rotation we have calculated an orientation difference of $35.4^{\circ}$ and $31.6^{\circ}$ for a sequence of three $60^{\circ}$ rotations with a statistical abundance ratio of $2: 1$. A detailed discussion on orientation differences and the corresponding relative intensities requires a complete analysis of rotation angle/axis pairs in terms of the "distribution function of the orientation differences" 15 and will be given elsewhere.

In summary, BKD patterns of diamond films have yielded detailed and quantitative information about the ori- entational arrangement of crystallites in the near-interface region on a $\mu \mathrm{m}$ scale. The quantitative output, the easy preparation, and the automatic measuring facilities qualify the OIM technique for systematic studies.

A high amount of orientation differences has been found which can be traced back to twin boundaries. We have shown that for the study of twin boundaries OIM somewhat combines the merits of TEM measuring the orientation difference of two neighboring points directly with the integrating character of methods like XRD to give statistical information on the orientation distribution of a large ensemble of single grains.

Because of the evolutionary growth process of diamond on $\mathrm{Si}(001)^{9}$ the film quality seems finally to be limited by the misalignment of the epitaxial nuclei with respect to the substrate. The possibility of the OIM technique to determine the orientations of the crystallites relative to the substrate directly at the interface offers a high potential for the optimization of diamond growth and, more speculating, for the optimization of the heteroepitaxial nucleation process. A further step will combine this technique with film etching and thus provide the information with high depth resolution. This should improve the current understanding of the mechanism of diamond growth and yield statistically significant data about the correlation between basic physical properties and the amount and types of grain boundaries in the film.

B.L.A. and K.K. gratefully acknowledge support under a Materials Research Group Award by the National Science Foundation No. (DMR-9001278). Also the software support by Th. Bollmeier is greatly appreciated.

${ }^{1}$ B. R. Stoner, G. H. M. Ma, S. D. Wolter, and J. T. Glass, Phys. Rev. B 45, 11067 (1992).

${ }^{2}$ W. Zhu, X. H. Wang, B. R. Stoner, G. H. M. Ma, H. S. Kong, M. W. H. Braun, and J. T. Glass, Phys. Rev. B 47, 6529 (1993).

${ }^{3}$ J. Narayan, J. Mater. Res. 5, 2414 (1990).

${ }^{4}$ D. Shechtman, J. L. Hutchison, L. H. Robins, E. N. Farabaugh, and A. Feldman, J. Mater. Res. 8, 473 (1993).

${ }^{5}$ B. R. Stoner, C. Koa, D. M. Malta, and R. C. Glass, Appl. Phys. Lett. 62, 2347 (1993).

${ }^{6}$ Ch. Wild, N. Herres, and P. Koidl, J. Appl. Phys. 68, 973 (1990).

${ }^{7}$ X. Jiang, C.-P. Klages, R. Zachai, M. Hartweg, and H.-J. Fuesser, Appl. Phys. Lett. 62, 3438 (1993).

${ }^{8}$ M. Schreck. R. Hessmer, S. Geier, B. Rauschenbach, and B. Stritzker, Diamond Relat. Mater. 3, 510 (1994).

${ }^{9}$ B. R. Stoner, S. R. Sahaida, J. P. Bade, P. Southworth, and P. J. Ellis, J. Mater. Res. 8, 1334 (1993).

${ }^{10}$ J. A. Venables and C. J. Harland, Philos. Mag. 27, 1193 (1973).

${ }^{11}$ D. J. Dingley and K. Baba-Kishi, in Scanning Electron Microscopy 1986 II (SEM AMF O'Hare, Chicago, 1986), p. 383; K. Z. Baba-Kishi and D. J. Dingley, J. Appl. Cryst. 22, 189 (1989).

${ }^{12}$ B. L. Adams, S. I. Wright, and K. Kunze, Metall. Trans. A 24, 819 (1993).

${ }^{13}$ D. J. Dingley (private communication).

${ }^{14}$ K. Kunze, S. I. Wright, B. L. Adams, and D. J. Dingley, Textures Microstruct. 20, 41 (1993).

${ }^{15}$ H.-J. Bunge, Texture Analysis in Materials Science (Cuvillier, Göttingen, 1993).

${ }^{16} \mathrm{~W}$. Bollmann, Crystal Defects and Crystalline Interfaces (Springer, New York, 1970).

${ }^{17}$ D. C. Handscomb, Can. J. Math. 10, 85 (1958). 